\title{
Topological Insulators with SU(2) Landau Levels
}

\author{
Yi Li, ${ }^{1,2}$ Shou-Cheng Zhang, ${ }^{3}$ and Congjun $\mathrm{Wu}^{1}$ \\ ${ }^{1}$ Department of Physics, University of California, San Diego, La Jolla, California 92093, USA \\ ${ }^{2}$ Princeton Center for Theoretical Science, Princeton University, Princeton, New Jersey 08544, USA \\ ${ }^{3}$ Department of Physics, Stanford University, Stanford, California 94305, USA
}

\begin{abstract}
We construct continuum models of 3D and 4D topological insulators by coupling spin- $\frac{1}{2}$ fermions to an $\mathrm{SU}(2)$ background gauge field, which is equivalent to a spatially dependent spin-orbit coupling. Higher dimensional generalizations of flat Landau levels are obtained in the Landau-like gauge. The 2D helical Dirac modes with opposite helicities and 3D Weyl modes with opposite chiralities are spatially separated along the third and fourth dimensions, respectively. Stable 2D helical Fermi surfaces and 3D chiral Fermi surfaces appear on open boundaries, respectively. The charge pumping in $4 \mathrm{D}$ Landau level systems shows quantized 4D quantum Hall effect.
\end{abstract}

PACS numbers: 73.43.Cd,71.70.Ej,73.21.-b

Time-reversal (TR) invariant topological insulators (TIs) have become a major research focus in condensed matter physics $[-3]$. Different from the 2D quantum Hall (QH) and quantum anomalous Hall systems which are topologically characterized by the first Chern number [48], time-reversal invariant TIs are characterized by the second Chern number in $4 \mathrm{D}[9,10]$ and the $\mathbb{Z}_{2}$ index in $2 \mathrm{D}$ and $3 \mathrm{D}[10-16]$. Various $2 \mathrm{D}$ and $3 \mathrm{D}$ TIs are predicted theoretically and identified experimentally exhibiting stable gapless 1D helical edge and 2D surface modes against TR invariant perturbations [13, 17 21]. Topological states have also been extended to systems with particle-hole symmetry and superconductors 22 24].

Most current studies of 2D and 3D TIs focus on Blochwave bands in lattice systems. The nontrivial band topology arises from spin-orbit (SO) coupling induced band inversions [13]. However, Landau levels (LL) are essential in the study of QH effects because their elegant analytical properties enable construction of fractional states. Generalizing LLs to high dimensions gives rise to TIs with explicit wave functions in the continuum, which would facilitate the study of the exotic fractional TIs. Efforts along this line were pioneered by Zhang and $\mathrm{Hu}$ [9]. They constructed LLs on the compact $S^{4}$ sphere by coupling fermions with the $\mathrm{SU}(2)$ monopole gauge potential, and various further developments appeared [25 29]. Two-dimensional TIs based on TR invariant LLs have also been investigated [12]. Two of the authors generalized LLs of Schrödinger fermions to high-dimensional flat space [30] by combining the isotropic harmonic potential and SO coupling. LLs have also been generalized to high dimensional Dirac fermions and parity-breaking systems 31, 32].

In all the above works, angular momentum is explicitly conserved; thus, they can be considered as LLs in the symmetric-like gauge. In 2D, LL wave functions in the Landau gauge are particularly intuitive: they are 1D chiral plane wave modes spatially separated along the transverse direction. The $\mathrm{QH}$ effect is just the $1 \mathrm{D}$ chiral anomaly in which the chiral current generated by the electric field becomes the transverse charge current. In this Letter, we develop high dimensional LLs with flat spectra as spatially separated helical Dirac or chiral Weyl fermion modes, i.e., the SU(2) Landau-like gauge. They are $3 \mathrm{D}$ and $4 \mathrm{D}$ TIs defined in the continuum possessing stable gapless boundary modes. To our knowledge, these are the simplest TI Hamiltonians constructed so far. Recently, there have been considerable interests of $2 \mathrm{D}$ topological band structures with approximate flat spectra. 33 35. Our Hamiltonians defined in the continuum possess exact flat energy spectra in $3 \mathrm{D}$ and $4 \mathrm{D}$, and are independent of the band inversion mechanism. For the 4D case, they exhibit the 4D quantum Hall effect [9, 10], which is a quantized nonlinear electromagnetic response related to the spatially separated $(3+1) \mathrm{D}$ chiral anomaly. Our methods can be easily generalized to arbitrary dimensions and also to Dirac fermions.

The 3D case. - We begin with the 3D TR invariant LL Hamiltonian for a spin- $\frac{1}{2}$ fermion as

$$
H_{L L}^{3 D}=\frac{\vec{p}^{2}}{2 m}+\frac{1}{2} m \omega_{s o}^{2} z^{2}-\omega_{s o} z\left(p_{x} \sigma_{y}-p_{y} \sigma_{x}\right),
$$

which couples the 1D harmonic potential in the $z$ direction and the 2D Rashba $\mathrm{SO}$ coupling through a $z$ dependent SO coupling strength. $H_{L L}^{3 D}$ possess translation symmetry in the $x y$ plane, TR and parity symmetries. Eq. (11) can be reformulated in the form of an $\mathrm{SU}(2)$ background gauge potential as $H_{L L}^{3 D}=\frac{1}{2 m}(\vec{p}-$ $\left.\frac{e}{c} \vec{A}\right)^{2}-\frac{1}{2} m \omega_{s o}^{2} z^{2}$, where $\omega_{s o}=|e G| / m c$, and $\vec{A}$ takes the Landau-like gauge as $A_{x}=G \sigma_{y} z, A_{y}=-G \sigma_{x} z$ and $A_{z}=0$. In Ref. [30], a symmetric-like gauge with $\overrightarrow{A^{\prime}}=G \vec{\sigma} \times \vec{r}$ is used, which explicitly preserves the $3 \mathrm{D}$ rotational symmetry. However, the $\mathrm{SU}(2)$ vector potentials $\overrightarrow{A^{\prime}}$ and $\vec{A}$ are not gauge equivalent. As shown below, the physical quantities of Eq. (1), such as density of states (DOS), are not 3D rotationally symmetric. Nevertheless, we will see below that these two Hamiltonians give rise to the same forms of helical Dirac surface modes, and thus they belong to the same topological class. A related Hamiltonian is also employed for studying electro- 
magnetic properties in superconductors with cylindrical geometry [36].

Equation (11) can be decomposed into a set of $1 \mathrm{D}$ harmonic oscillators along the $z$ axis exhibiting flat spectra, a key feature of LLs. We define a characteristic SO length scale $l_{s o}=\sqrt{\frac{\hbar}{m \omega_{s o}}}$. Each of the reduced 1D harmonic oscillator Hamiltonian is associated with a 2D helical plane wave state as $H^{z}\left(\vec{k}_{2 D}\right)=\frac{p_{z}^{2}}{2 m}+$ $\frac{1}{2} m \omega_{s o}^{2}\left[z-l_{s o}^{2} k_{2 D} \hat{\Sigma}_{2 D}\left(\hat{k}_{2 D}\right)\right]^{2}$, where $k_{2 D}=\left(k_{x}^{2}+k_{y}^{2}\right)^{\frac{1}{2}}$ and $\vec{k}_{2 D}=\left(k_{x}, k_{y}\right)$; the helicity operator is defined as $\hat{\Sigma}_{2 D}\left(\hat{k}_{2 D}\right)=\hat{k}_{x} \sigma_{y}-\hat{k}_{y} \sigma_{x}$. The $n$-th LL eigenstates are solved as

$$
\Psi_{n, \vec{k}_{2 D}, \Sigma}(\vec{r})=e^{i \vec{k}_{2 D} \cdot \vec{r}_{2 D}} \phi_{n}\left[z-z_{0}\left(k_{2 D}, \Sigma\right)\right] \otimes \chi_{\Sigma}\left(\hat{k}_{2 D}\right),(2)
$$

where $\vec{r}_{2 D}=(x, y) ; \chi_{\Sigma}\left(\hat{k}_{2 D}\right)$ are eigenstates of the helicity satisfying $\hat{\Sigma} \chi_{\Sigma}\left(\hat{k}_{2 D}\right)=\Sigma \chi_{\Sigma}\left(\hat{k}_{2 D}\right)$ with helicity eigenvalues $\Sigma= \pm 1 ; \phi_{n}\left[z-z_{0}\right]$ are the eigenstates of the $n$-th harmonic levels with the central positions located at $z_{0}$, and $z_{0}\left(k_{2 D}, \Sigma\right)=\Sigma l_{s o}^{2} k_{2 D}$. The energy spectra of the $n$-th LL is $E_{n}=\left(n+\frac{1}{2}\right) \hbar \omega_{s o}$, independent of $\vec{k}_{2 D}$ and $\Sigma$.
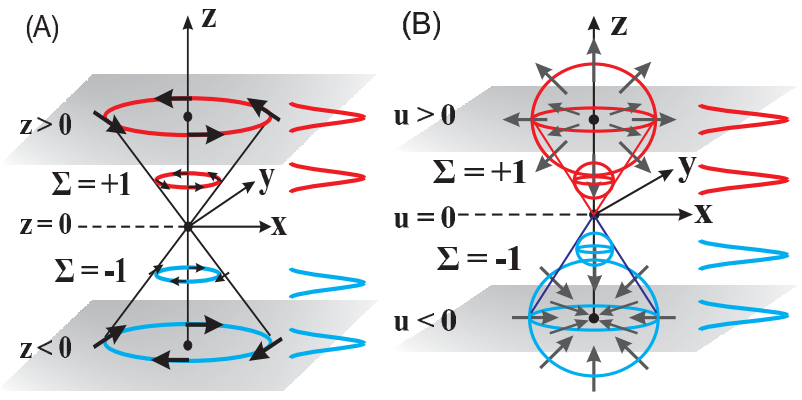

FIG. 1: $\quad 3 \mathrm{D}$ and $4 \mathrm{D}$ LLs for $H_{L L}^{3 D}$ and $H_{L L}^{4 D}$ as spatially separated $2 \mathrm{D}$ helical SO plane wave modes localized along the $z$ axis (A), and 3D Weyl modes localized along the $u$ axis (B), respectively. Their central locations are $z_{0}\left(\vec{k}_{2 D}, \Sigma\right)=\Sigma l_{s o}^{2} k_{2 D}$ and $u_{0}\left(\vec{k}_{3 D}, \Sigma\right)=\Sigma l_{s o}^{2} k_{3 D}$, respectively. Note that $2 \mathrm{D}$ Dirac modes with opposite helicities and the 3D ones with opposite chiralities are located at opposite sides of $z=0$ and $u=0$ planes, respectively.

In the 2D LL case, spatial coordinates $x$ and $y$ are noncommutative if projected to a given LL, say, the lowest LL (LLL). The LLL wave functions in the Landau gauge are 1D plane waves along the $x$ direction whose central $y$ positions linearly depend on $k_{x}$ as $y_{0}=l_{B}^{2} k_{x}$. These $1 \mathrm{D}$ modes with opposite chiralities are spatially separated along the $y$ direction. Consequently, the $x y$ plane can be viewed as the $2 \mathrm{D}$ phase space of a $1 \mathrm{D}$ system, in which $y$ plays the role of $k_{x}$. The momentum cutoff of the bulk states is determined by the system size along the $y$ direction as $\left|k_{x}\right|<\frac{L_{y}}{2 l_{B}^{2}}$. Right and left moving edge modes appear along the upper and lower boundaries perpendicular to the $y$ axis, respectively. These chiral edge modes cannot exist in purely 1D systems such as quantum wires.
Similarly, the 3D LL wave functions in Eq. (2) are spatially separated $2 \mathrm{D}$ helical plane waves along the $z$ axis. As shown in Fig. 1] (A), for states with opposite helicity eigenvalues, their central positions are shifted in opposite directions. Let us perform the LLL projection. Among the LLL states with good quantum numbers $\left(\vec{k}_{2 D}, \Sigma\right)$, it easy to check the following matrix elements $\left\langle\Psi_{0, \vec{k}_{2 D}, \Sigma}|z| \Psi_{0, \vec{k}_{2 D}^{\prime}, \Sigma^{\prime}}\right\rangle=\delta\left(\vec{k}_{2 D}, \vec{k}_{2 D}^{\prime}\right) \delta_{\Sigma, \Sigma^{\prime}} z_{0}\left(k_{2 D}, \Sigma\right)$. Therefore, we have $\left\langle\Psi_{0}^{a}|z| \Psi_{0}^{b}\right\rangle=\left\langle\Psi_{0}^{a}\right| \frac{l_{s o}^{2}}{\hbar}\left(p_{x} \sigma_{y}-\right.$ $\left.p_{y} \sigma_{x}\right)\left|\Psi_{0}^{b}\right\rangle$, where $\Psi_{0}^{a, b}$ are arbitrary linear superpositions of $\Psi_{0, \vec{k}_{2 D}, \Sigma}$ in the LLL. This proves that

$$
P z P=\frac{l_{s o}^{2}}{\hbar}\left(p_{x} \sigma_{y}-p_{y} \sigma_{x}\right),
$$

where $P$ is the LLL projection operator. Since the LLL states span the complete basis for the plane waves in the $x y$ plane, the projections of $x$ and $y$ in the LLL remain themselves. As a result, we obtain the following commutation relations after the LLL projection as

$$
[x, z]_{L L L}=i l_{s o}^{2} \sigma_{y},[y, z]_{L L L}=-i l_{s o}^{2} \sigma_{x},[x, y]_{L L L}=0 .
$$

Interestingly, the 3D LL states can be viewed as states in the $4 \mathrm{D}$ phase space of a $2 \mathrm{D}$ system (with $x$ and $y$ coordinates) augmented by the helicity structure, in which $|z|$ plays the role of the magnitude of the $2 \mathrm{D}$ momentum and the sign of $z$ corresponds to $\Sigma= \pm 1$. In fact, the momentum cutoff of the bulk states is exactly determined by the spatial size $L_{z}$ along the $z$ direction as

$$
k_{2 D}<k_{2 D}^{B} \equiv \frac{L_{z}}{2 l_{s o}^{2}},
$$

in which $-\frac{L_{z}}{2}<z<\frac{L_{z}}{2}$. Applying the open boundary condition along the $z$ direction, and periodic boundary conditions in the $x$ and $y$ directions with spatial sizes $L_{x}$ and $L_{y}$ respectively, we can easily count the total number of states to be $\mathcal{N}=\frac{L_{x} L_{y} L_{z}^{2}}{8 \pi l_{s o}^{4}}$. The $L_{z}^{2}$ dependence of $\mathcal{N}$ may seem puzzling for a $3 \mathrm{D}$ system, but expressing $L_{z}$ in terms of Eq. (5), we find $\mathcal{N}=\frac{1}{2 \pi} L_{x} L_{y}\left(k_{2 D}^{B}\right)^{2}$, which is the conventional state counting of a $2 \mathrm{D}$ system expressed in terms of the $4 \mathrm{D}$ phase space volume. It means that an effectively $4 \mathrm{D}$ density of states are squeezed into a $3 \mathrm{D}$ real space.

The topological property of this 3D LL system manifests through its helical surface spectra. Let us consider the upper boundary located at $z=z_{B}$ for $H_{L L}^{3 D}$. For simplicity, we only consider the LLL as an example. If $z_{B}>0$, the positive helicity states $\Psi_{0, \vec{k}_{2 D}, \Sigma=1}$ with $k_{2 D}>k_{2 D}^{B}=z_{B} l_{s o}^{-2}$ are confined at the boundary. The $1 \mathrm{D}$ harmonic potential associated with $\vec{k}_{2 D}$ and $\Sigma=1$ is truncated at $z=z_{B}$, and thus the surface spectra acquire dispersion. If we neglect the zero-point energy, the surface mode dispersion is approximated by $\frac{1}{2} m \omega_{s o}^{2}\left(k-k_{2 D}^{B}\right)^{2} l_{s o}^{4}$ with $k>k_{2 D}^{B}$. If the chemical potential $\mu$ lies above the LLL, it cuts the spectra at surface 
states with a Fermi wavevector $k_{f}>k_{2 D}^{B}$. The Fermi velocity is $v_{f} \approx m\left(k_{f}-k_{2 D}^{B}\right) \omega_{s o}^{2} l_{s o}^{4}$. The surface Hamiltonian is approximated as $H_{s f} \approx v_{f}(\vec{p} \times \vec{\sigma}) \cdot \hat{z}-\mu$ with an electronlike Fermi surface with $\Sigma=1$. In another case, if the upper boundary is located at $z_{B}<0$, then the negative helicity states $\Psi_{0, \vec{k}_{2 D}, \Sigma=-1}$ with $k_{2 D}<k_{2 D}^{B}$, and all the positive helicity states are pushed to the boundary as surface modes. Depending on the value of $\mu$, we can have a holelike Fermi surface with $\Sigma=-1$, a Dirac Fermi point, or an electronlike Fermi surface with $\Sigma=1$. Similarly, any other LL gives rise to a branch of gapless helical surface modes, and each filled bulk LL contributes one helical Fermi surface. For the lower boundary, the analysis is parallel to the above. Each filled LL gives rise to an electronlike helical Fermi surface with $\Sigma=-1$, or holelike with $\Sigma=1$. According to the standard $\mathrm{Z}_{2}$ classification, this system is topologically nontrivial if the Fermi energy cuts an odd number of Landau levels. So far, we have assumed the harmonic frequency and SO coupling frequency to be equal in Eq. (1). As explained in the Supplemental Material, although the equality of these two frequencies is essential for the spectra flatness, the $\mathbb{Z}_{2}$ topology does not require this equality.

The $4 D$ case The above procedure can be straightforwardly generalized to any higher dimension. For example, the 4D LL Hamiltonian is denoted as

$$
H_{L L}^{4 D}=\frac{p_{u}^{2}}{2 m}+\frac{1}{2} m \omega^{2} u^{2}+\frac{\vec{p}_{3 D}^{2}}{2 m}-\omega u \vec{p}_{3 D} \cdot \vec{\sigma},
$$

where $u$ and $p_{u}$ refer to the coordinate and momentum of the 4 th dimension, respectively, and $\vec{p}_{3 D}$ is the 3 -momentum in the $x y z$ space. Eq. 6] can be represented as $H_{L L}^{4 D}=\frac{1}{2 m} \sum_{i=1}^{4}\left(p_{i}-\frac{e}{c} A_{i}\right)^{2}-m \omega^{2} u^{2}$, where the $\mathrm{SU}(2)$ vector potential takes the Landau-like gauge with $A_{i}=G \sigma_{i} u$ for $i=x, y, z$ and $A_{u}=0$. Equation 6 preserves the translational and rotational symmetries in the $x y z$ space and TR symmetry. Similar to the 3D case, the 4D LL spectra and wave functions are solved by reducing Eq. (6) into a set of 1D harmonic oscillators along the $u$ axis as $H^{u}\left(\vec{k}_{3 D}\right)=\frac{p_{u}^{2}}{2 m}+\frac{1}{2} m \omega^{2}\left(u-l_{s o}^{2} k_{3 D} \hat{\Sigma}_{3 D}\right)^{2}$ where $k_{3 D}=\left(k_{x}^{2}+k_{y}^{2}+k_{z}^{2}\right)^{\frac{1}{2}}$ and $\hat{\Sigma}_{3 D}=\hat{k}_{3 D} \cdot \vec{\sigma}$. The LL wave functions are

$\Psi_{n, \vec{k}_{3 D}, \Sigma}(\vec{r}, u)=e^{i \vec{k}_{3 D} \cdot \vec{r}} \phi_{n}\left[u-u_{0}\left(k_{3 D}, \Sigma\right)\right] \otimes \chi_{\Sigma}\left(\vec{k}_{3 D}\right),(7)$

where, the central positions $u_{0}\left(k_{3 D}, \Sigma\right)=\Sigma l_{s o}^{2} k_{3 D} ; \chi_{\Sigma}$ are eigenstates of $3 \mathrm{D}$ helicity $\hat{\Sigma}_{3 D}$ with eigenvalues $\Sigma=$ \pm 1 . Inside each LL, the spectra are flat with respect to $\vec{k}_{3 D}$ and $\Sigma$. This realizes the spatial separation of the 3D Weyl fermion modes as shown in Fig. 1 (B). Similarly to the 3D case, after the LLL projection, we have the relation $P u P=(\vec{p} \cdot \vec{\sigma}) l_{s o}^{2} / \hbar$, and then the noncommutative relations among coordinates are

$$
\left[r_{i}, u\right]_{L L L}=i l_{s o}^{2} \sigma_{i}, \quad\left[r_{i}, r_{j}\right]_{L L L}=0,
$$

for $i=x, y, z$. The $4 \mathrm{D}$ LLs can be viewed as states of the $6 \mathrm{D}$ phase space of a 3D system: increasing the width along the $u$ direction corresponds to increasing the bulk momentum cutoff $k_{3 D}<k_{3 D}^{B} \equiv L_{u} /\left(2 l_{s o}^{2}\right)$. If the LLL is fully filled, the total number of states is given by $\mathcal{N}=\frac{L_{x} L_{y} L_{z} L_{u}^{3}}{24 \pi^{2} l_{s o}^{6}}$. Reexpressing $L_{u}=2 k_{3 D}^{B} l_{s o}^{2}$ we find $\mathcal{N}=\frac{1}{3 \pi^{2}} L_{x} L_{y} L_{z}\left(k_{3 D}^{B}\right)^{3}$, which is the conventional state counting of a $3 \mathrm{D}$ system expressed in terms of the $6 \mathrm{D}$ phase space volume. With an open boundary imposed along the $u$ direction, 3D helical Weyl fermion modes appear on the boundary.

Now let us consider the generalized 4D quantum Hall effects 10] as the nonlinear electromagnetic response of $(4+1) \mathrm{D}$ LL system to the external electric and magnetic (EM) fields, with $\vec{E} \| \vec{B}$ in the $x y z$ space. Without loss of generality, we choose the EM fields as $\vec{E}=E \hat{z}$ and $\vec{B}=B \hat{z}$, which are minimally coupled to the spin- $1 / 2$ fermion,

$$
H_{L L}^{4 D}(E, B)=-\frac{\hbar^{2}}{2 m} \nabla_{u}^{2}+\frac{1}{2} m \omega^{2}\left(u+i l_{s o}^{2} \vec{D} \cdot \vec{\sigma}\right)^{2},
$$

where $\vec{D}=\vec{\nabla}-i \frac{e}{\hbar c} \vec{A}_{e m}$. Here $\vec{A}_{e m}$ is the $U(1)$ magnetic vector potential in the Landau gauge with $A_{e m, x}=$ $0, A_{e m, y}=B x$ and $A_{e m, z}=-c E t$. We define $l_{B}=\sqrt{\frac{\hbar c}{e B}}$, where $e B>0$ is assumed.

The $\vec{B}$ field further reorganizes the chiral plane wave states inside the $n$-th $4 \mathrm{D}$ LL into a series of $2 \mathrm{D}$ magnetic LLs in the $x y$ plane. For the moment, let us set $E_{z}=0$. The eigenvalues $E_{n}=\left(n+\frac{1}{2}\right) \hbar \omega_{s o}$ remain the same as before without splitting, while the eigen-wave-functions are changed. We introduce a magnetic LL index $m$ in the $x y$ plane. For the case of $m=0$, the eigen-wave-functions are spin polarized as

$$
\begin{aligned}
\Psi_{n, m=0}\left(k_{y}, k_{z}\right) & =e^{i k_{y} y+i k_{z} z} \phi_{n}\left[u-u_{0}\left(k_{z}, m=0\right)\right] \\
& \otimes \chi_{m=0}\left[x-x_{0}\left(k_{y}\right)\right]
\end{aligned}
$$

where $x_{0}=l_{B}^{2} k_{y}$ and $\chi_{0}=\left[\phi_{0}\left(x-x_{0}\right), 0\right]^{T}$ is the zero mode channel of the operator $-i \vec{D} \cdot \vec{\sigma}$ with the eigenvalue of $\lambda_{0}=k_{z}$. The central positions of the $u$ direction harmonic oscillators are $u_{0}\left(k_{z}, m=0\right)=l_{s o}^{2} k_{z}$. For $m \geq$ 1 , the eigenmodes of $-i \vec{D} \cdot \vec{\sigma}$ come in pairs as

$$
\chi_{m, \pm}\left[x-x_{0}\left(k_{y}\right)\right]=\left(\begin{array}{c}
\alpha_{m, \pm} \phi_{m}\left(x-x_{0}\right) \\
\beta_{m} \phi_{m-1}\left(x-x_{0}\right)
\end{array}\right),
$$

where coefficients $\alpha_{m, \pm}=l_{B} k_{z} \pm \sqrt{l_{B}^{2} k_{z}^{2}+2 m}$, $\beta_{m}=-i \sqrt{2 m}$, and the eigenvalues are $\lambda_{m, \pm}=$ $\pm \sqrt{k_{z}^{2}+2 m l_{B}^{-2}}$. The corresponding eigen-wavefunctions are $\Psi_{n, m, \pm}\left(k_{y}, k_{z}\right)=e^{i k_{y} y+i k_{z} z} \phi_{n}[u-$ $\left.u_{0}\left(k_{z}, m, \pm\right)\right] \chi_{m, \pm}\left[x-x_{0}\left(k_{y}\right)\right]$, where the central positions $u_{0}\left(k_{z}, m, \pm\right)= \pm l_{\text {so }}^{2} \sqrt{k_{z}^{2}+2 m l_{B}^{-2}}$.

For the solutions of Eq. (10) with the same 4D LL index $n$, the 2D magnetic LLs with index $m=0$ are 


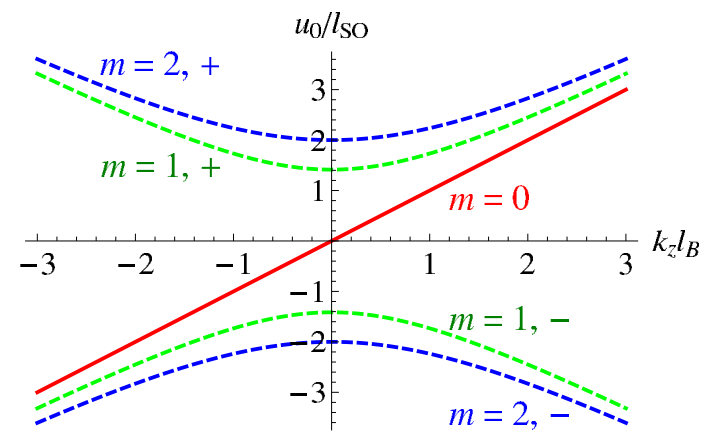

FIG. 2: The central positions $u_{0}\left(m, k_{z}, \nu\right)$ of the $4 \mathrm{D}$ LLs in the presence of the magnetic field $\vec{B}=B \hat{z}$. Only the branch of $m=0$ (shown in red) runs across the entire $u$ axis, which gives rise to quantized charge transport along $u$ axis in the presence of $\vec{E} \| \vec{B}$ as indicated in Eq. (12). This plot takes parameters $l_{\text {so }}=l_{B}$.

singled out. The central positions of states in this branch are linear with $k_{z}$, and thus run across the entire $u$ axis, while those of other branches with $m \geq 1$ only lie in one half of the space as shown in Fig. 2, After turning on $E_{z}, k_{z}$ is accelerated with time as $k_{z}(t)=k_{z}(0)+e E_{z} t / \hbar$, and thus the central positions $u_{0}\left(m=0, k_{z}\right)$ moves along the $u$ axis. Only the $m=0$ branch of the magnetic LL states contribute to the charge pumping which results in an electric current along the $u$ direction. Within the time interval $\Delta t$, the number of states with each filled $4 \mathrm{D}$ LL passing a cross section perpendicular to the $u$ axis is $N=\frac{L_{x} L_{y}}{2 \pi l_{B}^{2}} \frac{e E \Delta t L_{z}}{2 \pi}$, which results in the electric current density $\frac{e}{L_{x} L_{y} L_{z}} \frac{d N}{d t}$. If the number of fully filled $4 \mathrm{D}$ LLs is $n_{o c c}$, the total current density along the $u$ axis is

$$
j_{u}=n_{o c c} \alpha \frac{e}{4 \pi^{2} \hbar} \vec{E} \cdot \vec{B}
$$

where $\alpha=e^{2} /(\hbar c)$ is the fine-structure constant. This quantized non-linear electromagnetic response is in agreement with results from the effective theory [10] as the $4 \mathrm{D}$ generalization of the $\mathrm{QH}$ effect. If we impose open boundary conditions perpendicular to the $u$ direction, the above charge pump process corresponds to the chiral anomalies of Weyl fermions with opposite chiralities on the two 3D boundaries, respectively. Since they are spatially separated, the chiral current corresponds to the electric current along the $u$ direction.

Although the DOS of our 4D LL systems is different from the 4D TIs in the lattice system with translational symmetry [10], their electromagnetic responses obey the same Eq. (12). It is because that only the spin-polarized $m=0$ branch of LLs, $\Psi_{n, m=0}\left(k_{y}, k_{z}\right)$, is responsible for the charge pumping. For this branch, $B_{z}$ field quantizes the motion in the $x y$ plane so that the $x$ and $u$ coordinates play the role of $k_{y}$ and $k_{z}$, respectively. Consequently, the system can be viewed as the $4 \mathrm{D}$ phase space of coordinates $y$ and $z$, and scales uniformly as
$L_{x} L_{y} L_{z} L_{u}$ with the conventional thermodynamic limit of a $4 \mathrm{D}$ system.

The 3D LL Hamiltonian Eq. (1) can be realized in strained semiconductors by generalizing the method in Ref. [13] from 2D to 3D. For semiconductors with the zinc-blende structure, the $t_{2 g}$ components of the strain tensor generate $\mathrm{SO}$ coupling as

$$
\begin{aligned}
H_{s t n} & =-\lambda\left\{\epsilon_{x y}\left(p_{x} \sigma_{y}-p_{y} \sigma_{x}\right)+\epsilon_{y z}\left(p_{y} \sigma_{z}-p_{z} \sigma_{y}\right)\right. \\
& \left.+\epsilon_{z x}\left(p_{z} \sigma_{x}-p_{x} \sigma_{z}\right)\right\}
\end{aligned}
$$

where $\lambda=4 \times 10^{5} \mathrm{~m} / \mathrm{s}$ and $9 \times 10^{5} \mathrm{~m} / \mathrm{s}$ for GaAs and InSb, respectively [37]. The $z$-dependent Rashba SO coupling is induced by the strain component $\epsilon_{x y}$, which can be generated by applying the pressure along the [110] direction without inducing $\epsilon_{y z}$ and $\epsilon_{z x}[13,38]$. Furthermore, if the pressure linearly varies along the $z$ axis, such that $\epsilon_{x y}=g z$ where $g$ is the strain gradient, then we arrive at the SO coupling in Eq. (11) with the relation $\omega_{s o}=g \lambda$. A strain gradient of $1 \%$ over the length of $1 \mu \mathrm{m}$ leads to the LL gap $\hbar \omega=2.6 \mu \mathrm{eV}$ in GaAs. It corresponds to the temperature of $30 \mathrm{mK}$, which is accessible within the current low temperature technique. The strain gradient at a similar order has been achieved experimentally [13, 39]. The harmonic potential in Eq. (11) is equivalent to a standard parabolic quantum well along the $z$ axis [40], which is constructed by an alternating growth of GaAs and $\mathrm{Al}_{x} \mathrm{Ga}_{1-x}$ As layers. The harmonic frequency can be controlled by varying the thickness of different layers.

In conclusion, we have generalized LLs to $3 \mathrm{D}$ and $4 \mathrm{D}$ in the Landau-like gauge by coupling spatially dependent SO couplings with harmonic potentials. This method can be generalized to arbitrary dimensions by replacing the Pauli-matrices with the $\Gamma$ matrices in corresponding dimensions. These high dimensional LLs exhibit spatial separation of helical or chiral fermion modes with opposite helicities, which give rises to gapless helical or chiral boundary modes. The 4D LLs give rise to the quantized nonlinear electromagnetic responses as a spatially separated $(3+1) \mathrm{D}$ chiral anomaly. Many interesting open problems are left for future studies. For example, in the Supplemental Material, we present a preliminary effort on constructing of Laughlin wave functions in 4D LLs with spin polarizations. The generalizations of LLs to 3D and 4D Dirac fermions are also given there. A full study of the interaction effects on the high dimensional fractional topological states based on LLs will be investigated in future publications.

Y.L. and C.W. thank J. Hirsch, N.P. Ong, L. Sham, Z. Wang and Y.S. Wu for helpful discussions. Y.L. and C.W. are supported by the NSF DMR-1105945 and AFOSR FA9550-11-1-0067(YIP), and S.-C. Z. is supported by the NSF under Grant No. DMR-1305677. C.W. also acknowledges the support from the NSF of 
China under Grant No. 11328403. Y.L. thanks the Inamori Fellowship and the support at the Princeton Center for Theoretical Science.

[1] X.-L. Qi and S.-C. Zhang, Phys. Today 63, 33 (2010).

[2] X.-L. Qi and S.-C. Zhang, Rev. Mod. Phys. 83, 1057 (2011).

[3] M. Z. Hasan and C. L. Kane, Rev. Mod. Phys. 82, 3045 (2010).

[4] K. Klitzing, G. Dorda, and M. Pepper, Phys. Rev. Lett. 45, 494 (1980).

[5] D. J. Thouless, M. Kohmoto, M. P. Nightingale, and M. den Nijs, Phys. Rev. Lett. 49, 405 (1982).

[6] B. I. Halperin, Phys. Rev. B 25, 2185 (1982).

[7] M. Kohmoto, Ann. Phys. (N. Y.) 160, 343 (1985).

[8] F. D. M. Haldane, Phys. Rev. Lett. 61, 2015 (1988).

[9] S. Zhang and J. Hu, Science 294, 823 (2001).

[10] X.-L. Qi, T. L. Hughes, and S.-C. Zhang, Phys. Rev. B 78, 195424 (2008).

[11] C. L. Kane and E. J. Mele, Phys. Rev. Lett. 95, 226801 (2005).

[12] B. A. Bernevig and S. C. Zhang, Phys. Rev. Lett. 96, 106802 (2006).

[13] B. A. Bernevig, T. L. Hughes, and S. C. Zhang, Science 314, 1757 (2006).

[14] L. Fu and C. L. Kane, Phys. Rev. B 76, 045302 (2007).

[15] J. E. Moore and L. Balents, Phys. Rev. B 75, 121306 (2007).

[16] R. Roy, New J. Phys. 12, 065009 (2010).

[17] M. König et al., Science 318, 766 (2007).

[18] D. Hsieh et al., Nature (London) 452, 970 (2008).

[19] H. Zhang et al., Nat. Phys. 5, 438 (2009).

[20] Y. Xia et al., Nat. Phys. 5, 398 (2009).

[21] Y. L. Chen et al., Science 325, 178 (2009).

[22] X.-L. Qi, T. L. Hughes, S. Raghu, and S.-C. Zhang, Phys. Rev. Lett. 102, 187001 (2009).

[23] A. Kitaev, AIP Conf. Proc. 1134, 22 (2009).

[24] S. Ryu, A. Schnyder, A. Furusaki, and A. Ludwig, New J. Phys. 12, 065010 (2010).

[25] D. Karabali and V. Nair, Nucl. Phys. B 641, 533 (2002).

[26] H. Elvang and J. Polchinski, C. R. Acad. Sci. 4, 405 (2003).

[27] B. A. Bernevig, J. Hu, N. Toumbas, and S. C. Zhang, Phys. Rev. Lett. 91, 236803 (2003).

[28] K. Hasebe, Symmetry, Integrability Geom. Methods Appl. 6, (2010).

[29] J. M. Edge, J. Tworzydło, and C. W. J. Beenakker, Phys. Rev. Lett. 109, 135701 (2012).

[30] Y. Li and C. Wu, Phys. Rev. Lett. 110, 216802 (2013).

[31] Y. Li, K. Intriligator, Y. Yu, and C. Wu, Phys. Rev. B 85, 085132 (2012).

[32] Y. Li, X. Zhou, and C. Wu, Phys. Rev. B 85, 125122 (2012).

[33] E. Tang, J.-W. Mei, and X.-G. Wen, Phys. Rev. Lett. 106, 236802 (2011).

[34] K. Sun, Z. Gu, H. Katsura, and S. Das Sarma, Phys. Rev. Lett. 106, 236803 (2011).

[35] T. Neupert, L. Santos, C. Chamon, and C. Mudry, Phys. Rev. Lett. 106, 236804 (2011).

[36] J. E. Hirsch (private communication); Journal of Super- cond. 26, 2239 (2013).

[37] R. Ranvaud, H.-R. Trebin, U. Rössler, and F. H. Pollak, Phys. Rev. B 20, 701 (1979).

[38] G. Pikus and A. Titkov, Spin Relaxation under Optical Orientation in Semiconductors, Modern Problems in Condensed Matter Sciences Vol. 8, Chapter 3, (North-Holland, Amsterdam, 1984), p. 73.

[39] Q. Shen and S. Kycia, Phys. Rev. B 55, 15791 (1997).

[40] R. C. Miller, A. C. Gossard, D. A. Kleinman, and O. Munteanu, Phys. Rev. B 29, 3740 (1984).

\section{SUPPLEMENTAL MATERIAL}

In this supplemental material, we cover the classic equations of motion of the 3D Landau levels, further discussion of the topology class, the construction of Laughlin-type wavefunction, and the 3D Landau levels for relativistic fermions.

\section{CLASSICAL EQUATIONS OF MOTION}

The classical equations of motion for $H_{L L}^{3 D}$ (Eq. (1) in the main text) are derived as

$$
\begin{aligned}
\dot{\vec{r}} & =\frac{\vec{p}-\frac{e}{c} \vec{A}}{m}, & & \\
\dot{\vec{p}}_{2 D} & =0, & \dot{p}_{z} & =2 \omega(\vec{p} \times \vec{S})_{z}-m \omega^{2} z, \\
\dot{\vec{S}}_{2 D} & =-\frac{2 \omega}{\hbar} z S_{z} \vec{p}_{2 D}, & \dot{S}_{z} & =\frac{2 \omega}{\hbar} z \vec{S}_{2 D} \cdot \vec{p}_{2 D},
\end{aligned}
$$

where $\vec{S}_{2 D}$ and $\vec{S}_{z}$ are spin operators in the Heisenberg representation defined as $\vec{S}_{2 D}=\frac{1}{2} e^{i H_{L L}^{3 D} t}\left(\sigma_{x}, \sigma_{y}\right) e^{-i H_{L L}^{3 D} t}$ and $S_{z}=\frac{1}{2} e^{i H_{L L}^{3 D} t} \sigma_{z} e^{-i H_{L L}^{3 D} t}$.

If we choose the initial condition of spin $\vec{S}$ such that $S_{z, 0}=0$ and $\vec{S}_{2 D, 0} \perp \vec{p}_{2 D, 0}$, then $\vec{S}$ is conserved and lies in the $x y$ plane. The motion is reduced to a coplanar cyclotron one in the vertical plane perpendicular to $\vec{S}$. In other words, the orbital angular momentum and spin are locked, a feature from SO coupling.

\section{DISCUSSIONS ON THE MISMATCH OF THE TRAP AND SPIN-ORBIT FREQUENCIES}

In principle there should be two different frequencies in the 3D LL Hamiltonian Eq. 1 in the main text, the harmonic frequency denoted as $\omega_{T}$ below and the spinorbit frequency $\omega_{\text {so }}$. Below, we will explain that our $\mathbb{Z}_{2}$ analysis does not depend on the exact equality between $\omega_{T}$ and $\omega_{s o}$, and thus is generic. This equality is only important to maintain the spectra flatness which the usual $3 \mathrm{D}$ TIs do not possess.

Let us move back to a familiar example of the usual 2D LLs of quantum Hall systems in the Landau gauge. 
In a more general form, the Hamiltonian can also be represented in terms of two independent frequencies as

$$
\begin{aligned}
H_{2 D} & =\frac{\vec{p}^{2}}{2 m}+\frac{1}{2} m \omega_{T}^{2} y^{2}-\omega_{0} y p_{x} \\
& =\frac{p_{y}^{2}}{2 m}+\frac{1}{2} m \omega_{T}^{2}\left(y-\alpha l_{0}^{2} p_{x}\right)^{2}+\frac{1}{2 m}\left(1-\alpha^{2}\right) p_{x}^{2}(15)
\end{aligned}
$$

where $\alpha=\omega_{0} / \omega_{T}$ and $l_{0}^{2}=\hbar /\left(m \omega_{0}\right)$. The case of $\alpha=1$ corresponds to the usual 2D LL Hamiltonian

$$
H_{B}=\left(\vec{P}-\frac{e}{c} \vec{A}\right)^{2} / 2 m
$$

with $A_{x}=0, A_{y}=B x$ and $\omega_{0}=e B / m c$. Eq. (15) can also be viewed as Eq. (16) level with an extra harmonic potential along the $y$ axis with

$$
\Delta H=\frac{1}{2} m\left(\omega_{T}^{2}-\omega_{0}^{2}\right) y^{2} .
$$

In other words, $H_{2 D}=H_{B}+\Delta H . \omega_{T}$ needs to be larger than $\omega_{0}$, i.e. $\alpha \geq 1$, to ensure the spectra bounded from below. If $\alpha=1$, the spectra are exactly flat. If $\alpha<1$, the eigenstates are still plane-wave modes along with the good quantum number $k_{x}$ whose central positions are shifted according to $y_{0}\left(k_{x}\right)=\alpha l_{0}^{2} p_{x}$. The feature of the spatially separated chiral modes does not change. As a result, the topology remains the same as before, but their spectra become dispersive. If $\alpha$ is close to 1 , the dispersion is very slow. In this case, we can view this potential difference $\Delta H$ as a soft external potential imposing on the bulk Hamiltonian. The edge spectra of this quantum Hall system remains chiral, only the Fermi velocities are modified. This situation is met in mesoscopic quantum Hall systems. The topology of such a system is still characterized by the number of chiral edge modes.

Coming back to our case of 3D LL Hamiltonian Eq. (1) in the main text, the situation is very similar. As long as the time-reversal symmetry is preserved, and the energy scale of perturbations is much smaller than the Landau level gap, the $\mathbb{Z}_{2}$ topology is maintained. The concept of helicity due to spin-orbit coupling replaces chirality in the usual 2D Landau levels. When $\omega_{T}$ and $\omega_{s o}$ do not match, the Hamiltonian is expressed as

$$
\begin{aligned}
H_{L L}^{3 D} & =\frac{\vec{p}^{2}}{2 m}+\frac{1}{2} m \omega_{T}^{2} z^{2}-\omega_{s o} z\left(p_{x} \sigma_{y}-p_{y} \sigma_{x}\right), \\
& =\frac{p_{z}^{2}}{2 m}+\frac{1}{2} m \omega_{T}^{2}\left[z-\alpha l_{s o}^{2}\left(p_{x} \sigma_{y}-p_{y} \sigma_{x}\right)\right]^{2} \\
& +\frac{1}{2 m}\left(1-\alpha^{2}\right)\left(p_{x}^{2}+p_{y}^{2}\right),
\end{aligned}
$$

which gives rise to the following dispersive spectra as

$$
E_{n, \vec{k}, \pm}=\left(n+\frac{1}{2}\right) \hbar \omega_{T}^{2}+\left(1-\alpha^{2}\right) \frac{\hbar^{2}}{2 m}\left(k_{x}^{2}+k_{y}^{2}\right) .
$$

However, the eigenstates remains spatially separated helical 2D plane wave modes with good quantum numbers of $\left(k_{x}, k_{y}, \Sigma\right)$, whose central positions are shifted according to $z_{0}(\vec{k}, \Sigma)=\alpha \Sigma l_{s o}^{2} k$. As a result, the topology remains the same as before. In other words, the mismatch between $\omega_{T}$ and $\omega_{0}$ is equivalent to add the bulk Hamiltonian Eq. (1) with an external potential

$$
\Delta H(r)=\frac{1}{2} m\left(\omega_{T}^{2}-\omega_{s o}^{2}\right) z^{2}
$$

In the case of $\alpha$ close to 1 , this soft external potential imposes a finite sample size along the $z$ axis with the width $W^{2}<\hbar /(m \Delta \omega) \approx l_{s o}^{2} \omega_{T} / \Delta \omega$ where $\Delta \omega=\omega_{T}-$ $\omega_{0}$. Inside this region, $\Delta H$ is smaller than the LL gap, and the LL states are bulk states. The corresponding $2 \mathrm{D}$ wavevectors for these bulk states satisfy $k_{2 D}^{2}<l_{s o}^{-2} \frac{\omega_{T}}{\Delta \omega}$. Outside this width $W$, the LL states can be viewed as boundary modes with a fixed helicity. Again each branch of LL that cut the Fermi surface will contribute a helical surface Fermi surface, and thus the $\mathbb{Z}_{2}$ topology does not change, only the Fermi velocities of surface modes are affected.

\section{DELOCALIZED HELICAL MODES ON THE SIDE BOUNDARY}

In the main text, we have solved the helical surface states of the 3D LL Hamiltonian of Eq. (1) on the $x y$ plane. Each LL below the chemical potential contributes one helical Fermi surface. We further check the side surface below. According to the theorem proved in Ref. [4], as long as one surface exhibits a helical Fermi surface, all surfaces are topologically equivalent to odd numbers of channels of gapless delocalized helical modes. This theorem also applies to the side surface in our case in which the translational symmetry is absent along the $z$ direction. Following the reasoning in Ref. [4], let us consider two orthogonal surfaces of the $x y$ plane (top) and $x z$ plane (side) which intersect at the line of $x$ axis. Without loss of generality, we only consider the gap between the lowest and the second LLs. The translational symmetry along the $x$ axis is still maintained in spite of the open boundaries, and $k_{x}$ is conserved across the boundary between $x y$ and $x z$ surfaces. For a given energy $E$ lying inside this gap, it cuts surface states on the $x y$ plane with one helical iso-energy ring in momentum space centering around the origin $\left(k_{x}, k_{y}\right)=(0,0)$. Let us focus on the channel of modes with $k_{x}=0$. There are a pair of modes $\left(0, \pm k_{y}(E)\right)$ on the $x y$ surface, which cannot be scattered into each other at the intersection edge between $x y$ and $x z$ surfaces due to TR symmetry. Each of them has to continue on the $x z$ surface. According to the theorem in Ref. [4], the oddness of the number of TR pairs does not change on the $x z$ surface and cannot be localized even though the $z$ axis is not translational invariant. With varying the energy $E$ across the gap, we arrive at a branch of delocalized helical modes on the side surface of 
the $x z$ plane. On the other hand, for the case of $k_{x} \neq 0$, the modes in the $x y$ surface $\left(k_{x}, \pm k_{y}^{\prime}\right)$ with the energy $E$ are not TR partners, and thus they can be scattered into each other at the intersection edge. There is no guaranty that they need to continue on the side surface of $x z$ plane, or, the modes on the $x z$ plane with $k_{x} \neq 0$ are generally gapped and may be localized. In summary, on the side surface, although we cannot define Dirac cones due to the lack of translational symmetry, there do exist an odd number of helical delocalized modes.

\section{LAUGHLIN-TYPE WAVE FUNCTIONS}

These high dimensional LLs may provide a convenient platform for the further study of high dimensional interacting fractional topological states. The construction of the Laughlin-type wave functions for interacting fermions is difficult for these SO coupled high dimensional LL systems. Nevertheless, for the 4D case in the magnetic field, the LLL states with both $n=0$ and $m=0$ are spin-polarized, and their total DOS is finite as $\rho_{n=m=0}^{4 D}=\frac{1}{4 \pi^{2} l_{s o}^{2} l_{B}^{2}}$. Even though they are degenerate with other LLL states with $(n=0, m \neq 0)$, they are favored by repulsive interactions if they are partially filled.

The Laughlin wavefunction in the Landau gauge for the 2D LLs has been constructed in Ref. [1]. We generalize it to the $4 \mathrm{D}$ case, and define $w=e^{i \frac{x+i y}{L_{x}}}$ and $v=e^{i \frac{z+i u}{L_{z}}}$, then LL states are represented as $w^{h_{x}} v^{h_{z}}$ up to a Gaussian factor $e^{-\frac{u^{2}}{2 l_{s o}}-\frac{y^{2}}{2 l_{B}^{2}}}$. The Laughlin-type wavefunction can be constructed as

$$
\Psi\left(w_{1}, v_{1} ; \ldots ; w_{N_{x} N_{y}}, v_{N_{x} N_{y}}\right)=\left(\operatorname{det}\left[w_{i}^{h_{x}} v_{i}^{h_{z}}\right]\right)^{q}
$$

where $q$ is an odd number; $\operatorname{det}\left[w_{i}^{h_{x}} v_{i}^{h_{z}}\right]$ represents the Slater determinant for the fully filled single particle states $w^{h_{x}} v^{h_{z}}$ and $0 \leq h_{x} \leq N_{x}-1$ and $0 \leq h_{z} \leq N_{z}-1$. The study of the topological properties of Eq. 21 will be deferred to a later publication.

\section{HIGH DIMENSIONAL LLS OF DIRAC ELECTRONS}

The LL quantization based on 2D Dirac electrons have attracted a great deal of research attention since the discovery of graphene. Here we generalize LLs to the 3D and $4 \mathrm{D}$ relativisitc fermions, which are the square root problems to their Schrödinger versions in Eq. 1 and Eq.
6 For the $3 \mathrm{D}$ case, we have

$$
H_{L L, \text { Dirac }}^{3 D}=\frac{l_{s o} \omega_{s o}}{\sqrt{2}}\left[\begin{array}{cc}
0 & \vec{\sigma} \cdot \vec{p}+i \frac{z \hbar}{l_{s o}^{2}} \sigma_{z} \\
\vec{\sigma} \cdot \vec{p}-i \frac{z \hbar}{l_{s o}^{2}} \sigma_{z} & 0
\end{array}\right]
$$

Its square exhibits a diagonal block form with a supersymmetric structure as

$$
\frac{\left(H_{D i r a c}^{3 D}\right)^{2}}{\hbar \omega_{s o}}=\left(\begin{array}{cc}
H_{L L}^{3 D,+}-\frac{\hbar \omega_{s o}}{2} & 0 \\
0 & H_{L L}^{3 D,-}+\frac{\hbar \omega_{s o}}{2}
\end{array}\right)
$$

where $H_{L L}^{3 D,+}$ is just the 3D LL Hamiltonian given in Eq. 1 in the main text, and $H^{3 D,-}$ is given as

$$
H_{L L}^{3 D,-}=\frac{\vec{p}^{2}}{2 m}+\frac{1}{2} m \omega_{s o}^{2} z^{2}+\omega_{s o} z\left(p_{x} \sigma_{y}-p_{y} \sigma_{x}\right)
$$

The eigenvalues of Eq. (22) are $E_{ \pm n}= \pm \sqrt{n} \hbar \omega_{\text {so }}$. The eigen-wave-functions are constructed based on the eigenstates of $H_{L L}^{3 D, \nu= \pm}$ as

$$
\Psi_{ \pm n, \text { Dirac }}^{3 D}\left(\vec{k}_{2 D}, \Sigma\right)=\frac{1}{\sqrt{2}}\left(\begin{array}{c}
\Psi_{n, \vec{k}_{2 D}, \Sigma}^{+} \\
\pm \Psi_{n-1, \vec{k}_{2 D},-\Sigma}^{-}
\end{array}\right)
$$

where the eigenstate of $H_{L L}^{3 D,-}$ takes the form as

$$
\Psi_{n, \vec{k}_{2 D}, \Sigma}^{-}=e^{i \vec{k}_{2 D} \cdot \vec{r}_{2 D}} \phi_{n}\left[z+z_{0}\left(k_{2 D}, \Sigma\right)\right] \otimes \chi_{\Sigma}\left(\hat{k}_{2 D}\right) .
$$

The 0th LL states are Jackiw-Rebbi half-fermion modes with only the upper two components nonzero [2, 3].

The 4D LL Hamiltonian for Dirac Hamiltonian can be constructed as

$H_{L L, \text { Dirac }}^{4 D}=\frac{l_{s o} \omega_{s o}}{\sqrt{2}}\left[\begin{array}{cc}0 & \vec{\sigma} \cdot \vec{p}_{3 D}-i \frac{a_{u}}{l_{s o}} \\ \vec{\sigma} \cdot \vec{p}_{3 D}+i \frac{a_{u}^{\dagger}}{l_{s o}} & 0\end{array}\right]$

where $a_{u}=\frac{1}{\sqrt{2} l_{s o}}\left(u+i \frac{l_{s o}^{2}}{\hbar} p_{u}\right)$ is the phonon annihilation operator in the $u$ direction. The eigenvalues are still $E_{ \pm n}= \pm \sqrt{n} \hbar \omega_{s o}$, and the eigenstates are

$$
\Psi_{ \pm n, \text { Dirac }}^{4 D}\left(\vec{k}_{3 D}, \Sigma\right)=\frac{1}{\sqrt{2}}\left(\begin{array}{c}
\Psi_{n, \vec{k}_{3 D}, \Sigma} \\
\pm \Psi_{n-1, \vec{k}_{3 D}, \Sigma}
\end{array}\right)
$$

[1] E. H. Rezayi and F. D. M. Haldane, Phys. Rev. B 50, 17199 (1994).

[2] R. Jackiw and C. Rebbi, Phys. Rev. D 13, 3398 (1976).

[3] A. J. Niemi and G. W. Semenoff, Phys. Rep. 135, 99 (1986).

[4] Y. J. Jiang, F. Liu, F. Zhai, T. Low, and J. P. Hu, Phys. Rev. B 84, 205324 (2011). 